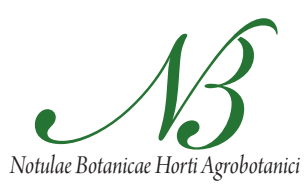

Cluj-Napoca

\title{
Stress-tolerant Wild Plants: a Source of Knowledge and Biotechnological Tools for the Genetic Improvement of Stress Tolerance in Crop Plants
}

\author{
Monica BOSCAIU', Pilar DONAT², Josep LLINARES², Oscar VICENTE* \\ ${ }^{1}$ Universitat Politécnica de València, Instituto Agroforestal Mediterráneo (IAM, UPV), CPI, \\ edificio8E, Camino de Veras/n, 46022 Valencia, Spain; mobosnea@eaf.upv.es \\ ${ }^{2}$ Universitat Politécnica de València, Instituto de Investigación para la Gestión Integrada de Zonas Costeras \\ (IGIC,UPV), Paranimf 1, 46730 Grau de Gandia, Valencia, Spain; jollipa@qim.upv.es \\ ${ }^{3}$ Universitat Politécnica de València, Instituto de Biologia Molecular y Celular de Plantas (IBMCP, UPV-CSIC), CPI, \\ edificio 8E, Camino de Vera s/n, 46022 Valencia, Spain; ovicente@ibmcp.upv.es (*corresponding author)
}

\begin{abstract}
Over the next few decades we must boost crop productivity if we are to feed a growing world population, which will reach more than $9 \times 10^{9}$ people by 2050; and we should do it in the frame of a sustainable agriculture, with an increasing scarcity of new arable land and of water for irrigation. For all important crops, average yields are only a fraction-somewhere between $20 \%$ and $50 \%$-of record yields; these losses are mostly due to drought and high soil salinity, environmental conditions which will worsen in many regions because of global climate change. Therefore, the simplest way to increase agricultural productivity would be to improve the abiotic stress tolerance of crops. Considering the limitations of traditional plant breeding, the most promising strategy to achieve this goal will rely on the generation of transgenic plants expressing genes conferring tolerance. However, advances using this approach have been slow, since it requires a deep understanding of the mechanisms of plant stress tolerance, which are still largely unknown. Paradoxically, most studies on the responses of plants to abiotic stress have been performed using stress-sensitive species-such as Arabidopsis thaliana-although there are plants (halophytes, gypsophytes, xerophytes) adapted to extremely harsh environmental conditions in their natural habitats. We propose these wild stress-tolerant species as more suitable models to investigate these mechanisms, as well as a possible source of biotechnological tools ('stress tolerance' genes, stress-inducible promoters) for the genetic engineering of stress tolerance in crop plants.
\end{abstract}

Keywords: abiotic stress, biotech crops, climate change, crop productivity, drought tolerance, salt tolerance, stress-tolerance genes

\section{Introduction}

There are at present $7 \times 10^{9}$ people in this planet, and over 900 million of them are undernourished; almost 6 million died of hunger during the first half of 2012: more than 30,000 every day. Devastating famines in underdeveloped African countries (most recently in the Horn of Africa and in the Sahel region, south of the Sahara desert) are often news-although rarely front-page news-in the media of industrialised countries. However, the world produces enough food to feed everyone on earth. Today's agriculture provides at least $2,700 \mathrm{Kcal}$ per person per day, $17 \%$ more than 30 years ago, despite the fact that world population has increased by $70 \%$ over the same period. This is mainly due to the huge increase in crop yields observed during the last few decades, as a consequence of several scientific and technical advances in agriculture: the development of new, more productive varieties for the most important crops, in which the so-called 'green revolution' was based, the massive use of agrochemicals (pesticides, herbicides, fertilizers) or, from 1996 on, the large-scale commercial cultivation of genetically modified-or 'biotech'-crops. Therefore, the principal cause of world hunger is not lack of food, but the extremely unequal distribution of food (money), in the world and within many poor countries. For example, the amount of money that would be required to solve the world hunger problem is only $30 \%$ of the value of the food wasted by USA consumers every year, or less than $80 \%$ of the spending in pet food in Europe and USA; almost $80 \%$ of all malnourished children of the world live in countries with food surpluses, and most of the hungriest nations export food to rich countries (Stop the Hunger, 2012; World Hunger Education Service, 2012). Obviously, apart from the political will to do it, there are many economic and logistic problems for a just worldwide distribution of food, but for the first time in man's history, we have the technical capacity to stop world hunger.

Unfortunately, the previous discussion may become a purely rhetoric question in the near future: at the present rates, the increases in agricultural production will not be able to cope with human population growth and by 2050 there will be no enough food for the ca. $9,2 \times 10^{9}$ people who are expected to be living on earth. Therefore, in the next decades we need to obtain a significantly larger 
324

amount of food from agriculture, but in conditions in which the most obvious approaches and the techniques successfully used in the past cannot be applied anymore. Specifically, any improvement of food production must be done in the frame of a sustainable agriculture, without further degradation of the environment or depletion of the natural resources our next generations should inherit.

\section{Improving crop productivity}

The most obvious and simplest way to increase food production, with the present methods and plant varieties, would be to enlarge the area used now to grow our crops; however, the land available for agriculture is actually decreasing, for several reasons. First, there is a change in land use in many parts of the world, mostly in some developing countries, due to a massive abandonment of rural areas, urban development close to big cities, industrial development in some areas, or tourism in others. More important, the spreading of desertification in many regions of the world brought about by global climate change (see below) is substantially contributing to decrease arable land. Moreover, there is not much additional land that could be used for agriculture, either because of its low fertility or due to its high ecological value (e.g., rain forests). Therefore, if the area of cultivated land cannot be increased significantly, crop productivity (i.e., yield per cultivated hectare) must be improved.

The zones in the world showing higher crop yields are those in arid and semi-arid regions cultivated under an irrigation regime; irrigated land represents only about $15 \%$ of the total cropland, but produces almost $40 \%$ of the world's food (Munns and Tester, 2008). The possibility to increase the irrigated area will be drastically limited by availability of water, which will become an ever scarcer resource, in part since it will be required for other uses (drinking water for people, water for industry), but also for the expected reduction of rainfall as a consequence-again-of climate change. To worsen the problem, these areas are progressively accumulating in the soil toxic ions dissolved in the irrigation water and suffering a 'secondary' (of anthropic origin) salinisation; this is causing the loss of more than 10 million ha of cropland every year (Owens, 2001). In non-irrigated land, the major problem for agriculture is drought, which also results in drastic yield reductions or even the complete loss of the crop. By 2050, up to $50 \%$ of the now available agricultural land may have been lost due to drought and soil salinity (Wang et al., 2003).

\section{Plant breeding for improved crop yields}

Yield improvement has always been one of the major objectives of traditional plant breeding, which best showed its potential with the successful development of high-yielding cereal varieties during the green revolution of the $20^{\text {th }}$ century. Classical breeding will, no doubt, contribute to the increase of crop productivity; however, due to its intrinsic limitations and the long time required to develop a new plant variety, the goal to feed humankind in the next decades will require much more efficient approaches.

As opposed to traditional breeding, genetic engineering provides the techniques to generate new (transgenic) plant varieties with the desired traits, in a rapid and controlled manner, by transfer of specific genes. Genetically modified-or 'biotechnological'-crops have been grown commercially for more than 15 years, with a continuous increase of their cultivation area-from $1.7 \times 10^{6}$ ha in 1996 to $148 \times 10^{6}$ ha in 2010 (James, 2011). Practically all this cropland is used for cultivation of only three major foodcrops-soybean, maize and rapeseed-plus cotton. This 'first generation' of GM plants was designed to improve some agronomic traits: herbicide tolerance (HT) and insect resistance (IR), and also, to a much lesser extent, virus resistance (VR); the present trend is to combine several transgenes in the same plant ('stacked traits'). It is clear that these GM crops have allowed obtaining higher average yields; for example, only in 2007, their combined production gains amounted to 32 million tons, which would have required 10 million ha of additional land to be produced by conventional crops (James, 2009). For each of these species, transgenic varieties account for a significant percentage of the global cultivated cropland, from 23\% for rapeseed, about $30 \%$ for maize and more than $80 \%$ in the case of soybean; these percentages are much higher in the USA: $91 \%$ for soybean (100\% in Argentina), $85 \%$ for maize (as in Brazil) or $88 \%$ for rapeseed ( $95 \%$ in Canada) [data from 2010 (James 2011)].

Therefore, another simple way to increase crop productivity, with the present varieties, would be to extend worldwide cultivation of the three major transgenic food crops-or, at least, of GM maize and rapeseed, since there is no much room left for increasing cultivation of transgenic soybean. In addition, there are some major GM crops already developed, but not yet commercialised, such as Monsanto's HT wheat, which could be cultivated in large areas in a relatively short time. China has recently started to grow its own Bt rice. Finally, there are a number of other minor, HT, IR or VR biotech crops (alfalfa, squash, pepper, papaya, etc) which are now cultivated in a very small area $(<0.1 \%$ of global GM cropland) and could easily be grown at a much larger level. Nevertheless, the yield improvements of transgenic crops, as compared with their conventional counterparts, are not extremely high; farmers get higher income from GM crops, but not only because of the increased productivity, but also by a more stable production, reduction in labour, and significantly lower energy costs. Therefore, even combining all the above-mentioned strategies, the expected production gains with the already available GM varieties will be short of the required increase in food availability. Biotech crops with new traits must be developed to reach this goal. 


\section{Abiotic stress and crop productivity}

There is much variability in the productivity of a particular cultivated species, depending on a multitude of factors: type of soil, environmental conditions, use of fertilizers, presence of weeds, pests or pathogens but, for all crops, average yields are only a fraction of record yields. These differences are mostly due to abiotic stress conditions (water or salt stress, heavy metals, flooding, cold, high temperatures, UV irradiation, etc.) which produce losses ranging from about $50 \%$, for sugar beet or potato for example, to more than $80 \%$, as it is the case for sorghum or wheat. Drought and high soil salinity, in particular, are the major causes reducing crop yields worldwide (Boyer, 1982).

Therefore, there is a wide margin for the increase of crop productivity by breeding for stress tolerance, and especially if drought and salt tolerance of the major crops could be improved. Availability of drought tolerant crops will allow their cultivation in dry lands, without depending on irrigation water or at least requiring less water, a commodity which is becoming increasingly scarce, as indicated before; they could even help to recover for agriculture abandoned land, where growing conventional crops is not profitable due to the low yields obtained. Similarly, salt tolerant crops would have a significant influence on future crop productivity in irrigated land, since they could maintain high and stable yields, despite soil salinisation, could be grown using brackish water for irrigation-thus saving good-quality fresh water for human consumption and other uses-or help to reclaim agricultural land already lost due to salinisation. Finally, both stress tolerant crops might be cultivated in marginal soils, also contributing to extend the area available for agriculture.

The foreseeable effects of global climate change-an increase of average temperatures, a general reduction of rainfall, alteration of the normal seasonal weather patterns, a higher frequency, intensity and duration of extreme meteorological phenomena, such as floods, droughts, 'heat waves' etc. (DB Climate Change Advisors, 2009)-will worsen the plants' stressful environmental conditions in many regions, thus making even more urgent the development of stress tolerant crops. As to the methods to be employed, here again generation of transgenic plants expressing 'stress-tolerance' genes is the most promising approach, since classical plant breeding has been until now relatively unsuccessful in obtaining stress resistant varieties, except for a few particular cases.

\section{Mechanism of abiotic stress tolerance}

The isolation and characterization of genes conferring tolerance to stress by expression in GM crops requires the previous, in-depth understanding of the mechanisms plants use as a response to stress, which-together with the academic interest of the topic-has stimulated the study of these mechanisms over the last two decades. These studies have revealed a series of basic, conserved stress response pathways, apparently used by all plants-tolerant as well as sensitive-which are activated at the cellular level in response to different types to abiotic stress, and include: $i$ ) the control of water transport, ion transport and ion homeostasis, to prevent cellular dehydration and to maintain osmotic balance, including the compartmentalization of toxic ions in the vacuole and the synthesis and accumulation of compatible solutes or 'osmolytes' in the cytosol; these osmolytes have additional functions as 'osmoprotectants', directly stabilizing proteins and cellular structures under dehydration conditions and protecting the cell against oxidative stress as scavengers of 'reactive oxygen species' (ROS); ii) synthesis of specific protective proteins, such as osmotine, heat-shock proteins, 'late-embryogenesis abundant' (LEA) proteins, etc. and iii) synthesis of antioxidant compounds (GSH, flavonoids and other phenolics, carotenoids, vitamins $\mathrm{C}$ and $\mathrm{E}$, etc.) and activation of enzymatic antioxidant systems (superoxide dismutase, catalase, ascorbate peroxidase, glutathione peroxidase, glutathione reductase, etc.), induced in response to oxidative stress generated either directly (e.g., by ozone or high UV irradiation) or secondarily by other stressful environmental conditions (Ashraf, 2009; Hussain et al., 2008; Munns, 2002; Vinocur and Altman, 2005; Wang et al., 2003; Zhu, 2001).

\section{Searching for 'Stress Tolerance' genes}

It seemed logical to assume that stress tolerance mechanism in plants would be based on the above-mentioned stress response pathways, although it is far from clear the relative contribution of the different responses for a given species in each particular set of environmental conditions. Therefore, it was expected that overexpression of genes involved in these response mechanisms would increase the stress tolerance of transgenic plants. In fact, some positive results have been obtained by expression of genes encoding ion transporters, enzymes of osmolyte biosynthesis, specific 'anti-stress' proteins or antioxidant enzymes, which conferred, indeed, variable levels of tolerance to drought, salinity, high temperatures and/or other abiotic stresses. In our laboratory, two $A$. thaliana genes, encoding splicing factors of the SR-like family, were isolated based on the tolerance to $\mathrm{LiCl}$ conferred by their expression in yeast, and were shown as well to increase salt ( $\mathrm{LiCl}$ and $\mathrm{NaCl}$ ) tolerance when overexpressed in transgenic Arabidopsis plants (Forment et al., 2002). More recently, we have confirmed that these 'SR-like' proteins also confer a marked drought-resistance phenotype to the transgenics (Bourgon et al., 2007). These results suggest that RNA processing-or RNA metabolism, in general-is very sensitive to abiotic stress, and provide new possible targets for engineering tolerance in plants. In most cases, however, these studies have been carried out using model species, especially Arabidopsis thaliana, and there are few data on the genetic engineering of crop plants for abiotic stress tolerance. 
326

Concerning the commercial development of salt-tolerant crops, it appears to be still far away. Promising results were described more than ten years ago-by expressing the AtNHXI gene, encoding the Arabidopsis $\mathrm{Na}^{+} / \mathrm{H}^{+}$vacuolar antiporter, in tomato (Zhang and Blumwald, 2001)but could not be reproduced by other groups. An alternative approach, based on cell type-specific alteration of sodium transport, to exclude this toxic cation from the shoots, may result more effective in increasing salt tolerance (Møller et al., 2009), but these experiments have been performed only in Arabidopsis, and it remains to be seen whether they can be extended to crops.

The first drought tolerant crop, on the contrary, has just been commercially launched. Monsanto, in collaboration with BASF, has developed a GM maize variety transformed with bacterial genes encoding RNA chaperones (Castiglioni et al., 2008). After going through all the regulatory process and field trials, the company obtained approval in USA and Canada, and this year (2012), for the first time, is growing the crop in the more droughtprone U.S. states of Nebraska and Kansas. However, the expected increments in yield (at least for this ' $1^{\text {st }}$ generation' drought-resistant GM-maize) are very modest, of no more than 10\%; some improvement is expected with more advanced 'versions' of the crop and by introducing the trait in other, more drought-tolerant cultivars obtained by classical breeding. By 2017, drought tolerant maize will probably be available for Sub Saharan Africa. Promising results have also been obtained in field trials of drought tolerant wheat in Australia, with the best GM lines yielding 20\% more than their conventional counterparts, and without apparent yield penalty under irrigation (GMO Safety, 2008).

Are the right models to study stress tolerance being used?

At present, we have a fairly good knowledge of the mechanisms used by plants to respond to different abiotic stresses as a result of more than 20 years of intensive research on this specific topic. However, there are not yet stress tolerant, high yielding crops growing in our fields. The slow advance in obtaining these urgently needed plant varieties could be due, at least in part, to a certain confusion regarding the related concepts of 'stress responses' and 'stress tolerance', and to the selection of inappropriate experimental models.

Most biochemical and molecular studies on the plant responses to abiotic stress mentioned before have been carried out using the model plant Arabidopsis thaliana-or, to a much lesser extent, some crop species. Also, the putative stress-tolerance genes available at present have been isolated from the same species. The advantages of Arabidopsis as the model in plant molecular biology are well known and, in fact, its use to investigate the stress response pathways cannot be criticised, as these general responses appear to be essentially conserved in all plant species. A priori, however, Arabidopsis does not seem to be the most appropri- ated species to investigate stress tolerance mechanisms simply because it is not stress-tolerant, but rather sensitive to even relatively mild stress condition-as a large fraction of wild plants and all crops.

However, there are plants naturally adapted to environmental stress conditions-which could often be extremely harsh-in their natural habitats. They include, for example, the halophytes, salt-tolerant plants which grow and complete their biological cycle in a wide variety of ecosystems with high soil salinity (> $200 \mathrm{mM} \mathrm{NaCl}$ ) (Flowers and Colmer, 2008): littoral or interior salt marshes, swamps, saline deserts, dunes or cliffs by the sea. The xerophytes are plants adapted to arid and semi-arid regions, with low levels of rainfall and soil humidity. The gypsophytes are plants living in gypsum soils, which combine salt and drought stress. In addition, all these habitats are generally poor in nutrients. Many of these species, and the corresponding habitats, have been subjected to botanical and ecological studies; in some cases, their stress responses have been studied at the physiological or biochemical levels, generally under laboratory or greenhouse conditions. In any case, our knowledge of the molecular mechanisms of stress tolerance in these wild plants is still very limited.

Assuming that all plants use indeed the same stress response pathways, it is clear that in most species (including the Arabidopsis model), the activation of those stress responses in general do not lead to stress tolerance, while they do in wild plants adapted to particular types of stress in nature. Therefore, the mechanisms of response to abiotic stress of wild tolerant plants must be more efficient than those operating in non-tolerant species, although both may share the same molecular basis. In other words, the differences observed in the responses to stress of tolerant and non-tolerant species are of a quantitative rather than a qualitative nature.

\section{An underutilised genetic resource?}

In our opinion, stress tolerant wild plants, such as halophytes, xerophytes and gypsophytes, represent useful complementary models, at present underutilised, for the investigation of stress tolerance mechanisms at the physiological, biochemical and molecular levels. Presumably, the use of these wild tolerant species will contribute to increase our knowledge in this field, providing relevant information, complementary to that obtained from the stress sensitive models commonly used until now. It would be also very interesting to carry out comparative studies using plant species with similar genetic background but different degrees of tolerance, for example related tolerant and sensitive taxa of the same genus.

To explain the quantitative differences in the responses to environmental stress of tolerant and sensitive species, it could be assumed that certain proteins-encoded by 'stress tolerance' genes-playing essential roles in the mechanisms of tolerance have a higher intrinsic activity in the tolerant species than the homologous proteins from the sensi- 
tive one. These proteins could include, among others, ion transporters of the plasma membrane or the tonoplast, enzymes involved in osmolyte biosynthesis, enzymatic antioxidant systems or proteins regulating the expression or activity of any of them. On the other hand, the differences in the response could also be due to differences in the level of expression of the corresponding genes, either because of the relative strength of their promoters or because of their regulatory mechanisms. For example, the expression of a particular gene could be stress-inducible in a tolerant species, but not in a related non-tolerant taxon.

Therefore, stress-tolerant wild plants also represent a possible source of new, more efficient biotechnological tools for the genetic improvement of stress tolerance in crop plants: genes conferring higher levels of tolerance by overexpression in transgenic plants (as compared to homologous genes isolated from stress sensitive species), or stress-regulated promoters which could be used for the controlled expression of any putative stress tolerance gene.

\section{References}

Ashraf M (2009). Biotechnological approach of improving plant salt tolerance using antioxidants as markers. Biotech Adv 27:84-93.

Bourgon L, Amorós B, Naranjo MA, Vicente O (2007). Drought and salt tolerance conferred by overexpression of splicing factors in transgenic plants. Bulletin USAMV-CN 64:1-6.

Boyer JS (1982). Plant productivity and environment. Science 218:443-448.

Castiglioni P, Warner D, Bensen RJ, Anstrom DC, Harrison J, Stoecker M, Abad M, Kumar G, Salvador S, D’Ordine R, Navarro S, Back S, Fernandes M, Targolli J, Dasgupta S, Bonin C, Luethy MH, Heard JE (2008). Bacterial RNA chaperones confer abiotic stress tolerance in plants and improved grain yield in maize under water-limited conditions. Plant Physiol 147:446-455.

DB Climate Change Advisors (2009). Investing in agriculture: Far-reaching challenge, significant opportunity. An asset management perspective. (Deutsche Bank report). Whitepaper available online at: http://www.dbcca.com/ research.

Flowers TJ, Colmer TD (2008). Salinity tolerance in halophytes. New Phytol 179:945-963.
Forment J, Naranjo MA, Roldán M, Serrano R, Vicente O (2002). Expression of Arabidopsis SR-like splicing proteins confers salt tolerance to yeast and transgenic plants. Plant J 30:511-519.

GMO Safety (2008). Drought-tolerant wheat: "Promising results". Available online at: http://www.gmosafety.eu/en/ news/.

Hussain TM, Chandrasekhar T, Hazara M, Sultan Z, Saleh BK, Gopal GR (2008). Recent advances in salt stress biology-a review. Biotechnol Mol Biol Rev 3:8-13.

James C (2009). Global Status of Commercialized Biotech/GM Crops: 2008. ISAAA Brief No. 39. ISAAA: Ithaca, NY.

James C (2011). Global Status of Commercialized Biotech/GM Crops: 2010. ISAAA Brief No. 42. ISAAA: Ithaca, NY.

Møller IS, Gilliham M, Jha D, Mayo GM,. Roy SJ, Coates JC, Haseloff J, Tester M (2009). Shoot $\mathrm{Na}^{+}$exclusion and increased salinity tolerance engineered by cell type-specific alteration of $\mathrm{Na}^{+}$transport in Arabidopsis. Plant Cell 21:2163-2178.

Munns R (2002). Comparative physiology of salt and water stress. Plant Cell Environ 25:239-250.

Munns R, Tester M (2008). Mechanisms of salinity tolerance. Annu Rev Plant Biol 59:651-681.

Owens S (2001). Salt of the earth. Genetic engineering may help to reclaim agricultural land lost due to salinisation. EMBO Rep 2:877-879.

Stop the Hunger (2012). World hunger statistics updated in real time. Available online at: http://www.stopthehunger.com.

Vinocur B, Altman A (2005). Recent advances in engineering plant tolerance to abiotic stress: achievements and limitations. Curr Opin Biotechnol 16:123-132.

Wang W, Vinocur B, Altman A (2003). Plant responses to drought, salinity and extreme temperatures: towards genetic engineering for stress tolerance. Planta 218:1-14.

World Hunger Education Service (2012). World hunger and poverty facts and statistics. Available on line at: http://www. worldhunger.org.

Zhang HX, Blumwald E (2001). Transgenic salt-tolerant tomato plants accumulate salt in foliage but not in fruit. Nat Biotechnol 19:765-768.

Zhu J-K (2001). Plant salt tolerance. Trends Plant Sci 6:66-71. 